\title{
Controlling uncertain Lü system using linear feedback
}

\author{
Jinhu L $\ddot{u ̈}^{\mathrm{a}, *}$, Junan $\mathrm{Lu}^{\mathrm{b}}$ \\ a Institute of Systems Science, Academy of Mathematics and System Sciences, Chinese Academy of Sciences, \\ Beijing 100080, PR China \\ ${ }^{\mathrm{b}}$ School of Mathematics and Statistics, Wuhan University, Wuhan 430072, PR China
}

Accepted 7 October 2002

\begin{abstract}
Lü attractor is a new chaotic attractor, which connects the Lorenz attractor and Chen attractor and represents the transition from one to the other. An effective observer is produced to identify the unknown parameters of Lü system. Moreover, a linear feedback control strategy is proposed for controlling uncertain Lü system. Numerical simulations show the effectiveness and feasibility of the proposed controllers.
\end{abstract}

(c) 2002 Elsevier Science Ltd. All rights reserved.

\section{Introduction}

The research on controlling chaos has seen a dramatic increase over the last decade [1,2]. Chaos control, in a broader sense, can be divided into two categories: one is to suppress the chaotic dynamical behavior and the other is to generate or enhance chaos in nonlinear systems [1,2] (known as chaotification or anti-control of chaos).

Recently, different techniques and methods [1-9] have been proposed to achieve chaos control. For instance, OGY method [3], linear state space feedback [4], backstepping design method [5], differential geometric method [6], inverse optimal control [7], sampled-data feedback control [8] and adaptive control [9], among many others [1-3].

However, for uncertain dynamical systems, many of the aforementioned methods will fail, where uncertain means that some parameters of system are unknown. An interesting problem in chaos control is how to achieve linear control of uncertain chaotic systems. This problem involves the identification of the unknown parameters and the techniques of chaos control. This letter applies the linear feedback techniques to controlling chaos in Lü system [10-12]. The effective observers are provided to identify the unknown parameters of Lü system. Then the simple feedback functions are designed for controlling Lü system. Also, the proposed method can enable the controlled Lü system to approach any desired points or periodic orbits. Computer simulations are given for illustrating the effectiveness of the approach.

\section{System description}

In 1963, Lorenz found the first canonical chaotic attractor [13], which has just been mathematically confirmed to exist [14]. In 1999, Chen found another similar but topologically not equivalent chaotic attractor [15], as the dual of the Lorenz system, in a sense defined by Vaněček and Čelikovský [16]: The Lorenz system satisfies the condition $a_{12} a_{21}>0$ while Chen system satisfies $a_{12} a_{21}<0$, where $a_{12}, a_{21}$ are the corresponding elements in the constant matrix $A=\left(a_{i j}\right)_{3 \times 3}$ for the linear part of the system. Very recently, Lü and Chen found a new chaotic system [10-12], bearing the name of the Lü system (as named for convenience by others [17,18]), which satisfies the condition $a_{12} a_{21}=0$, thereby bridging the gap between the Lorenz and Chen attractors $[11,12]$. The new chaotic system is described by

\footnotetext{
${ }^{*}$ Corresponding author. Tel.: +86-10-6265-1362; fax: +86-10-6254-1689.

E-mail address: lvjinhu@amss.ac.cn (J. Lü).
} 


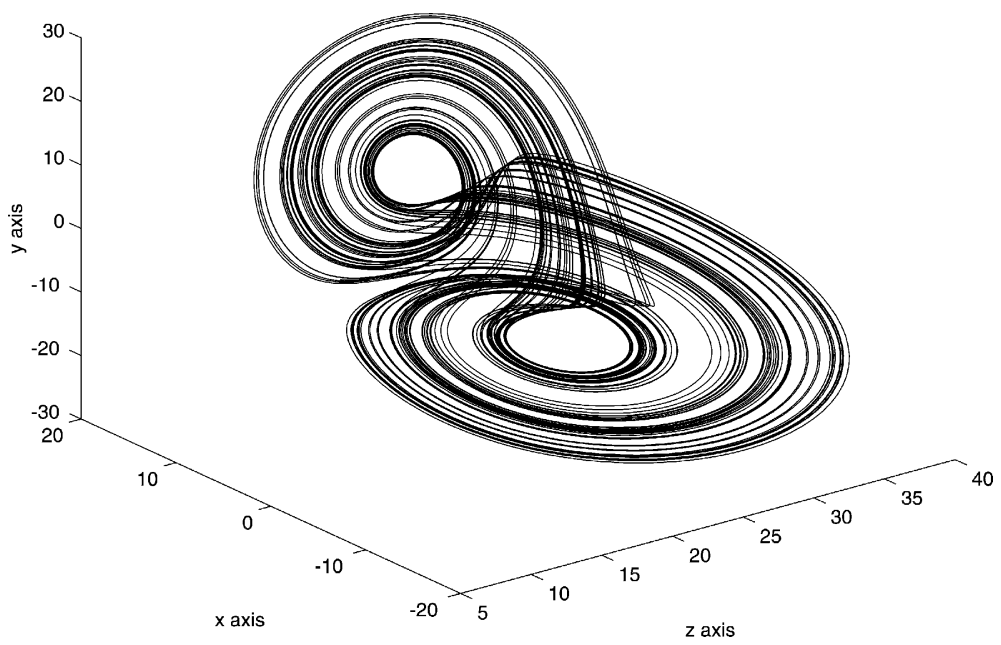

Fig. 1. Lü chaotic attractor.

$$
\left\{\begin{array}{l}
\dot{x}=a(y-x), \\
\dot{y}=-x z+c y, \\
\dot{z}=x y-b z
\end{array}\right.
$$

which has a chaotic attractor as shown in Fig. 1 when $a=36, b=3, c=20$.

It is very interesting to develop control techniques which can drive a strange attractor of uncertain system not only to an equilibrium point but also a periodic orbit. For convenience, we assume that the parameters $b$ and $c$ of system (1) are unknown. Moreover, we add two control inputs to the first and second equations, respectively. Thus the controlled system becomes:

$$
\left\{\begin{array}{l}
\dot{x}=a(y-x)+u_{1}, \\
\dot{y}=-x z+c y+u_{2}, \\
\dot{z}=x y-b z .
\end{array}\right.
$$

\section{Identification of the unknown parameters}

In this section, the observers are provided to identify the unknown parameters $b$ and $c$ of the Lü system.

Definition. For a system $\Sigma$, reconstruct a new system $\Sigma_{g}$, and use the observable variable of original system $\Sigma$, such as output vector $y$ and input vector $u$, as its input signal, make its output signal $\hat{x}(t)$ equivalent with the status vector $x(t)$ of $\Sigma$ under a certain index. Then the system $\Sigma_{g}$ is called as observer.

For the uncertain Lü system, since parameter $c$ is unknown, then we do not know the relevant dynamical information about the parameter $c$. However, we can attain the output vector $(x, y, z)$. In the following, we devise an observer that can identify the unknown parameter $c$. Note that $c$ is a constant, thus

$$
\dot{c}=0 .
$$

Since the unknown parameter $c$ can act as a status variable, the system (1) can be augmented by Eq. (3). From system (1), we get $c y=x z+\dot{y}$. Then we can design the following observer

$$
\dot{\hat{c}}=-F(y) y \hat{c}+F(y)(x z+\dot{y}),
$$

where $F(y)$ is a gain function. Let

$$
e=c-\hat{c},
$$


then according to Eqs. (3) and (4), we get

$$
\dot{e}(t)=\dot{c}-\dot{\hat{c}}=-F(y) y e(t) \text {. }
$$

Thus we can select a gain function $F(y)$ so that the system

$$
\dot{e}(t)+F(y) y e(t)=0,
$$

is exponentially asymptotically stable for all $y$. That is, for $t \rightarrow \infty, \hat{c}(t)$ converges to $c(t)$ with exponential rate. Obviously, the gain function $F(y)=k y$ satisfies above condition. Then we have

$$
\dot{e}(t)+k y^{2} e(t)=0,
$$

where $k>0$ determines the convergence rate. In fact, $\dot{y}$ is hard to observe, so the observer (4) is of no use. We introduce an auxiliary variable

$$
\delta_{1}=\hat{c}-P(y),
$$

where $P(y)$ is a design function and satisfies $F(y)=\mathrm{d} P(y) / \mathrm{d} y$.

From (8), we get

$$
\dot{\delta}_{1}=\dot{\hat{c}}-\frac{\mathrm{d} P(y)}{\mathrm{d} y} \dot{y}=-F(y) y \delta_{1}+F(y)(x z-y P(y))
$$

and

$$
\hat{c}=\delta_{1}+P(y) .
$$

Obviously, if the design function $P(y)$ can drive the system

$$
\dot{e}(t)+\frac{\mathrm{d} P(y)}{\mathrm{d} y} y e(t)=0,
$$

exponentially asymptotically stable, then for $t \rightarrow \infty, \hat{c}(t)$ will converge to $c(t)$ with exponential rate. Therefore, the observers (9) and (10) can identify the unknown parameter $c$ of system (1), where $F(y)$ is a gain function and $P(y)$ a design function satisfying $\mathrm{d} P(y) / \mathrm{d} y=F(y)$.

Note that the observers (9) and (10) only rely on the second equation of system (1). That is, when the structures of the first and third equations of system (1) or the parameters $a$ and $b$ are varied, the results of the identification are not changed. Hence, the observers have strong robustness. If $P(y)=k y^{2} / 2$, the observers become

$$
\left\{\begin{array}{l}
\dot{\delta}_{1}=-k y^{2} \delta_{1}+k x y z-\frac{k^{2} y^{4}}{2}, \\
\hat{c}=\delta_{1}+\frac{k y^{2}}{2} .
\end{array}\right.
$$

Similarly, in order to identify parameter $b$, we devise the following observers

$$
\left\{\begin{array}{l}
\dot{\delta}_{2}=-k \delta_{2}+k^{2} \ln z+\frac{k x y}{z}, \\
\hat{b}=\delta_{2}-k \ln z
\end{array}\right.
$$

\section{Linear feedback control}

In this section, we present our main results concerning a new and simple control technique, which can drive the trajectories of the controlled uncertain Lü system to approach any target points or periodic orbits.

Firstly, we apply the observers (11) and (12) to identify the unknown parameters $b$ and $c$ of system (1). Therefore, we get $b=\hat{b}$ and $c=\hat{c}$.

For any $x_{0}$ near the chaotic attractor, according to the ergodic property of chaotic orbits, Lü system can reach the point $x=x_{0}$, but will not stay at $x=x_{0}$ without further control. In order to stabilize Lü system at $x=x_{0}$, the control inputs must satisfy the stable condition:

$$
\frac{\mathrm{d} x}{\mathrm{~d} t}=\frac{\mathrm{d} y}{\mathrm{~d} t}=\frac{\mathrm{d} z}{\mathrm{~d} t}=0 .
$$


Let the control inputs $u_{1}$ and $u_{2}$ be both linear, in the following form

$$
\left\{\begin{array}{l}
u_{1}=-a y-a\left|x_{0}\right|\left(x-x_{0}-\operatorname{sign}\left(x_{0}\right)\right), \\
u_{2}=x-(\hat{c}+1) y
\end{array}\right.
$$

thus the controlled system (2) becomes

$$
\left\{\begin{array}{l}
\dot{x}=-a x-a\left|x_{0}\right|\left(x-x_{0}-\operatorname{sign}\left(x_{0}\right)\right), \\
\dot{y}=x-x z-y \\
\dot{z}=x y-\hat{b} z
\end{array}\right.
$$

Obviously, the system (14) has a unique equilibrium point $S_{0}\left(x_{0}, \hat{b} x_{0} /\left(\hat{b}+x_{0}^{2}\right), x_{0}^{2} /\left(\hat{b}+x_{0}^{2}\right)\right)$.

It can be easily verified that the Jacobian matrix $A\left(S_{0}\right)$ of system (14) is

$$
A\left(S_{0}\right)=\left(\begin{array}{ccc}
-a-a\left|x_{0}\right| & 0 & 0 \\
\frac{\hat{b}}{\hat{b}+x_{0}^{2}} & -1 & -x_{0} \\
\frac{\hat{b} x_{0}}{\hat{b}+x_{0}^{2}} & x_{0} & -\hat{b}
\end{array}\right),
$$

whose characteristic equation is

$$
\left(\lambda+a+a\left|x_{0}\right|\right)\left[\lambda^{2}+(\hat{b}+1) \lambda+\hat{b}+x_{0}^{2}\right]=0 .
$$

Since the three characteristic roots have negative real part, the unique equilibrium point $S_{0}$ is stable.

Combining (11) and (12), we attain the control law of the controlled system (2) with unknown parameters $b$ and $c$

$$
\left\{\begin{array}{l}
u_{1}=-a y-a\left|x_{0}\right|\left(x-x_{0}-\operatorname{sign}\left(x_{0}\right)\right), \\
u_{2}=x-(\hat{c}+1) y \\
\dot{\delta}_{1}=-k y^{2} \delta_{1}+k x y z-\frac{k^{2} y^{4}}{2} \\
\hat{c}=\delta_{1}+\frac{k y^{2}}{2} \\
\dot{\delta}_{2}=-k \delta_{2}+k^{2} \ln z+\frac{k x y}{z} \\
\hat{b}=\delta_{2}-k \ln z
\end{array}\right.
$$

where $k>0$ is a control constant.

Therefore, under the feedback control inputs (16), Lü system with unknown parameters $b$ and $c$ will approach the target point $S_{0}$.

Similarly, in order to guide the controlled system (2) to reach an arbitrary point $x=x_{1}, y=y_{1}$, let the linear controller be

$$
\left\{\begin{array}{l}
u_{1}=-a y-a\left|x_{1}\right|\left(x-x_{1}-\operatorname{sign}\left(x_{1}\right)\right), \\
u_{2}=x-(\hat{c}+1) y-|d|\left(y-y_{1}-\operatorname{sign}(d)\right),
\end{array}\right.
$$

where $d=-x_{1}+y_{1}+\left(x_{1}^{2} y_{1} / \hat{b}\right)$.

So that the controlled system (2) becomes

$$
\left\{\begin{array}{l}
\dot{x}=-a x-a\left|x_{1}\right|\left(x-x_{1}-\operatorname{sign}\left(x_{1}\right)\right), \\
\dot{y}=x-x z-y-|d|\left(y-y_{1}-\operatorname{sign}(d)\right), \\
\dot{z}=x y-\hat{b} z
\end{array}\right.
$$

Obviously, the system (18) has unique equilibrium point $S_{1}\left(x_{1}, y_{1}, x_{1} y_{1} / \hat{b}\right)$. And the corresponding characteristic equation of system (18) is

$$
\left(\lambda+a+a\left|x_{1}\right|\right)\left[\lambda^{2}+(1+\hat{b}+|d|) \lambda+\hat{b}+x_{1}^{2}+\hat{b}|d|\right]=0,
$$

then the unique steady state $S_{1}$ is stable.

Considering (11) and (12), we get the control technique of the controlled system (2) with unknown parameters $b$ and $c$ 


$$
\left\{\begin{array}{l}
u_{1}=-a y-a\left|x_{1}\right|\left(x-x_{1}-\operatorname{sign}\left(x_{1}\right)\right), \\
u_{2}=x-(\hat{c}+1) y-|d|\left(y-y_{1}-\operatorname{sign}(d)\right), \\
\dot{\delta}_{1}=-k y^{2} \delta_{1}+k x y z-\frac{k^{2} y^{4}}{2} \\
\hat{c}=\delta_{1}+\frac{k y^{2}}{2} \\
\dot{\delta}_{2}=-k \delta_{2}+k^{2} \ln z+\frac{k x y}{z} \\
\hat{b}=\delta_{2}-k \ln z
\end{array}\right.
$$

where $k>0$ is a control constant.

Hence, under the action of feedback control input (19), Lü system with unknown parameters $b$ and $c$ can quickly approach to target point $S_{1}$.

Next, in order to guide the trajectories of the controlled uncertain Lü system to approach any target periodic orbits, we assume that $x_{1}$ and $y_{1}$ are periodic functions. Let

$$
x_{1}=r \cos \omega t, \quad y_{1}=r \sin \omega t
$$

and

$$
\left\{\begin{array}{l}
u_{1}=-a y-a r|\cos \omega t|(x-r \cos \omega t-\operatorname{sign}(\cos \omega t)) \\
u_{2}=x-(\hat{c}+1) y-|d|(y-r \sin \omega t-\operatorname{sign}(d)) \\
\dot{\delta}_{1}=-k y^{2} \delta_{1}+k x y z-\frac{k^{2} y^{4}}{2} \\
\hat{c}=\delta_{1}+\frac{k y^{2}}{2} \\
\dot{\delta}_{2}=-k \delta_{2}+k^{2} \ln z+\frac{k x y}{z} \\
\hat{b}=\delta_{2}-k \ln z
\end{array}\right.
$$

where $d=\left(r^{3} \sin 2 \omega t \cos \omega t / 2 \hat{b}\right)+r \sin \omega t-r \cos \omega t$, and $k>0$ is a control constant.

Under the feedback control inputs (21), we can easily prove that the uncertain Lü system will approach to the target periodic orbit

$$
x_{1}=r \cos \omega t, \quad y_{1}=r \sin \omega t, \quad z=\frac{r^{2} \sin 2 \omega t}{2 \hat{b}} .
$$

This kind of periodic orbit is called "periodic harmonic oscillation" which usually is not embedded in a chaotic attractor. It is just a nice regular periodic motion, harmonic motion, which has no relation with chaos in general.

In practice, the feedback controller (21) can easily be realized. We can devise a outer oscillator, which produces periodic sine signal. Using the periodic signal of this oscillator as control input signals, we can guide the Lü system with unknown parameters $b$ and $c$ to stabilize at the periodic orbit (22).

\section{Simulations}

In this section, numerical simulations are given to verify the effectiveness of the observers (11), (12) and the control applicability of the proposed control laws (16), (19) and (21).

In all simulations, we assume $a=36, k=0.8$, initial conditions $x(0)=1.0, y(0)=-0.5, z(0)=1.5$, and the time step size $h=0.001 \mathrm{~s}$. The fourth order Runge-Kutta method is used to solve differential equations, such as (14) and (18).

Fig. 2 shows the effectiveness of the observers (11) and (12). We investigate the effectiveness of observers under the action of without external force and external force $f=5$ when $t=45 \mathrm{~s}$, respectively.

Fig. 3 shows the better control applicability of the proposed control technique (16) for different $x_{0}$. It indicates that the controlled system (2) can reach target point $\left(x_{0}, \hat{b} x_{0} /\left(\hat{b}+x_{0}^{2}\right), x_{0}^{2} /\left(\hat{b}+x_{0}^{2}\right)\right)$ within $1.7 \mathrm{~s}$ for two different $x_{0}$.

Fig. 4 displays the better control applicability of the provided control method (19) for different $x_{1}, y_{1}$. It indicates that the controlled system (2) can reach target point $\left(x_{1}, y_{1}, x_{1} y_{1} / \hat{b}\right)$ within $2.2 \mathrm{~s}$ for two different $x_{1}, y_{1}$.

Fig. 5 shows the better control applicability of the presented control law (21) for different $r, \omega$. It indicates that:

- For different $r, \omega$, the controlled system (2) can quickly approach periodic orbits within $2 \pi / \omega \mathrm{s}$. The period is $T=2 \pi / \omega$, which is not distinctly dependent on orbits;

- With the increasing of parameter $r$, the controlled system (2) will more quickly approach to target periodic orbits. It may be the reason that the feedback control signal becomes more stronger with the increasing of parameter $r$; 

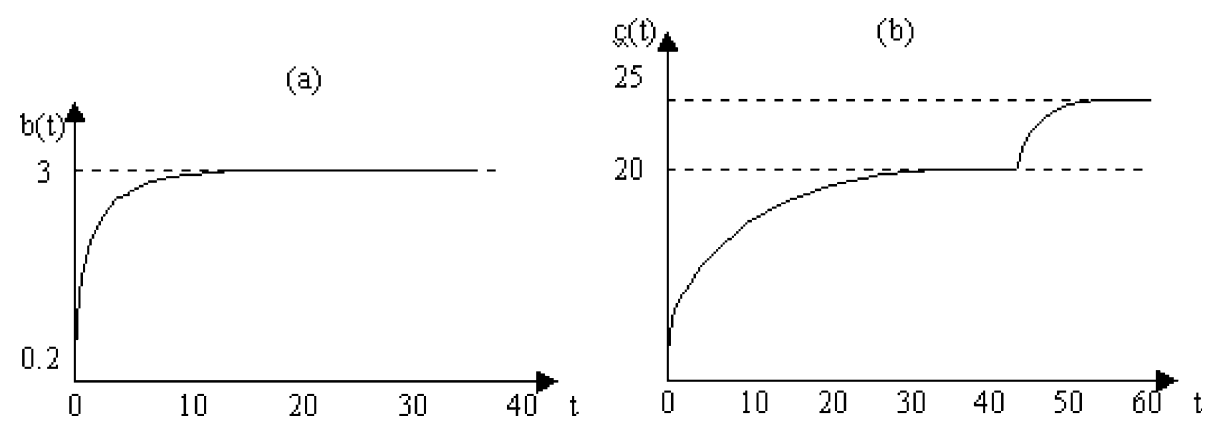

Fig. 2. The identification results of Lü system: (a) the identification of $b$ under without external force; (b) the identification of $c$ under external force $f=5$ when $t=45 \mathrm{~s}$.
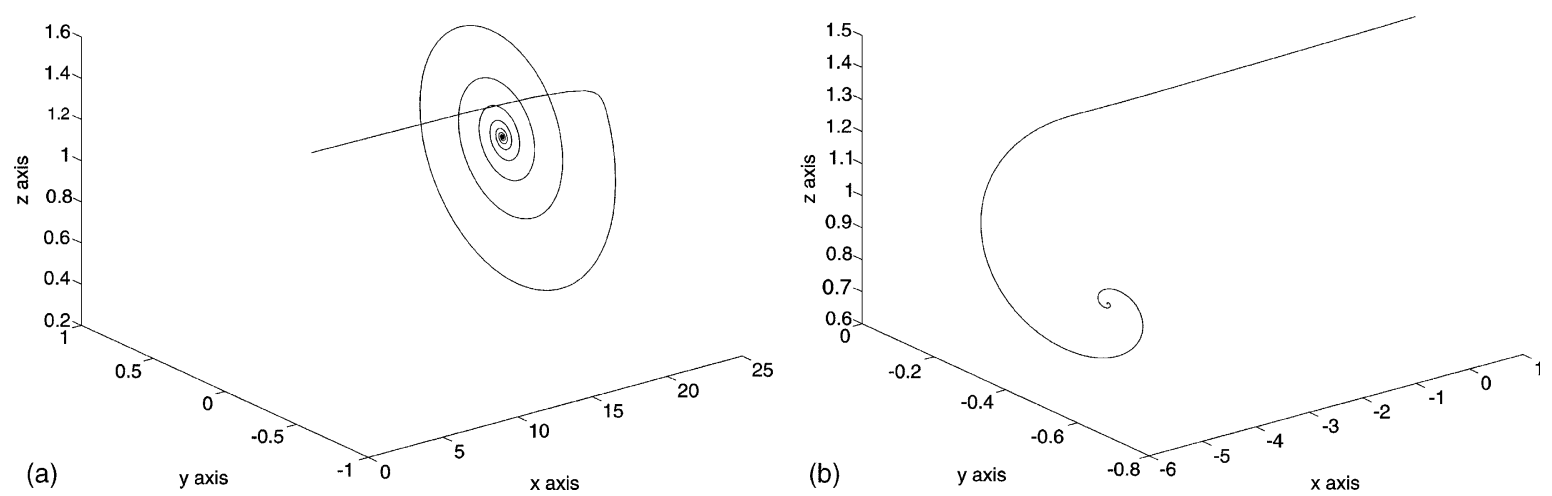

Fig. 3. Approach target points: (a) $x_{0}=20, S_{0}(20,0.1489,0.9926)$; (b) $x_{0}=-5, S_{0}(-5,-0.5357,0.8929)$.
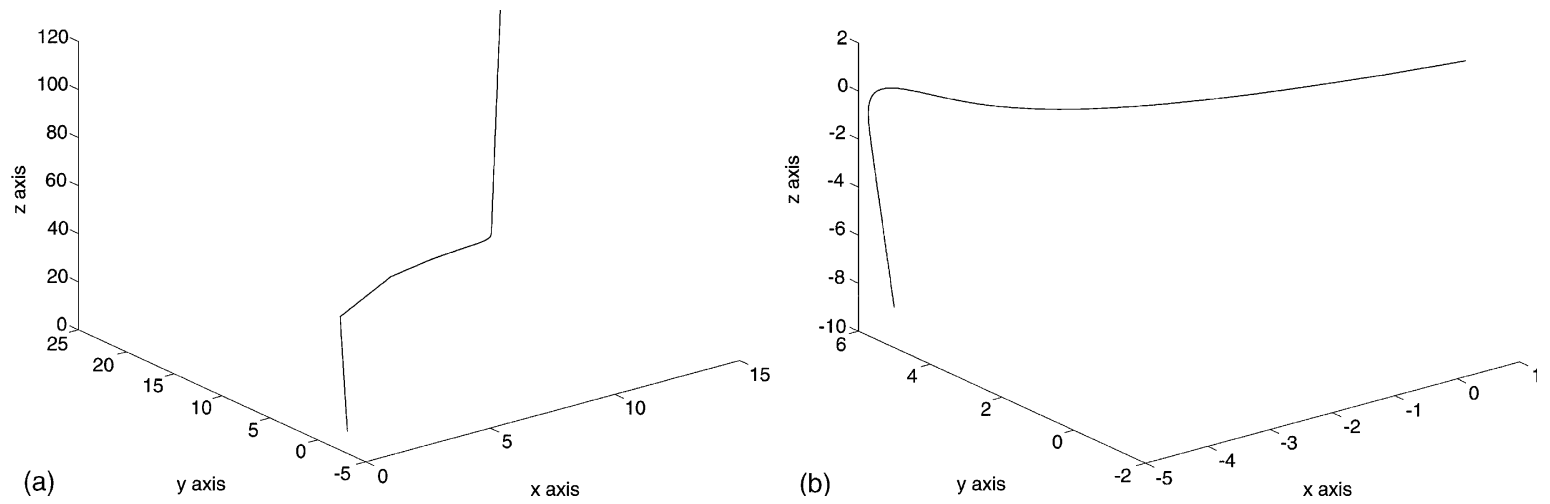

Fig. 4. Approach target points: (a) $x_{1}=15, y_{1}=20, S_{0}(15,20,100)$; (b) $x_{1}=-5, y_{1}=5, S_{0}(-5,5,-8.3333)$.

- For $0.15<r<1$, the controlled system (2) can stabilize at periodic orbits. For $r \rightarrow 0$, the controlled system (2) is unstable at periodic orbit. It may be the reason that the feedback control signal becomes relatively weaker;

- For $r>1$ and $0.03<\omega<120$, the projected orbits on the $x-y$ plane will evolve from round to ellipse.

Numerical simulations confirm our theoretical analysis. However, a little difference between the theoretical analysis and numerical simulation, we think, may be caused by the relatively stronger feedback control signal. 

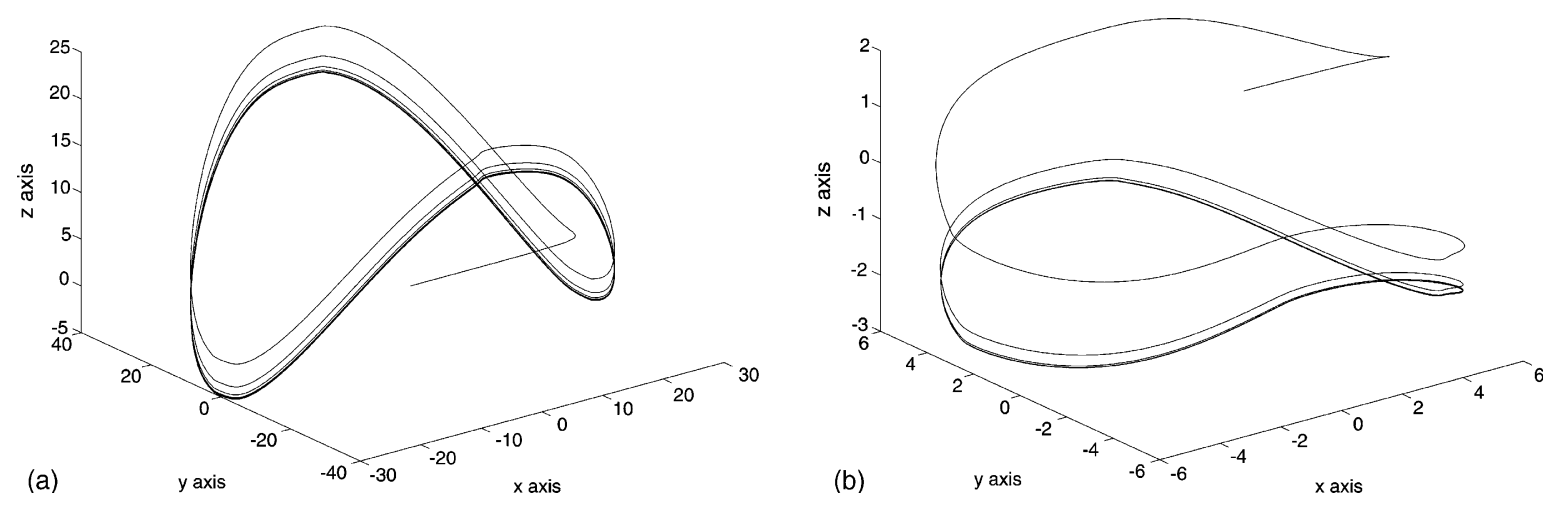

Fig. 5. Approach target periodic orbits: (a) $r=30, \omega=20$; (b) $r=6, \omega=12$.

\section{Conclusion}

An effective control technique has been produced to guide the trajectories of uncertain Lü system to approach any target points or periodic orbits. Compared with existing other chaos control laws, this controller can identify unknown parameters and approach any desired targets or periodic orbits in a short time. Furthermore, the targets of controlling chaos can be deduced beforehand. Especially, this technique combines the identification of unknown parameters with linear feedback control method to control uncertain chaotic system.

It should be pointed out that although this paper focuses mainly on the control of uncertain Lü system, we believe that the better control applicability of the proposed control law can justify the practical application of the proposed method to some other complex uncertain dynamical systems.

\section{Acknowledgement}

The authors thank Professor Guanrong Chen for offering some papers and suggestions.

\section{References}

[1] Chen G, Dong X. From chaos to order: methodologies, perspectives and applications. Singapore: World Scientific; 1998.

[2] Lü J, Lu J, Chen S. Chaotic time series analysis and its application. China: Wuhan University Press; 2002.

[3] Ott E, Grebogi C, Yorke JA. Controlling chaos. Phys Rev Lett 1990;64:1196-9.

[4] Chen G, Dong X. On feedback control of chaotic continuous-time systems. IEEE Trans Circ Syst 1993;40:591-601.

[5] Lü J, Zhang S. Controlling Chen's chaotic attractor using backstepping design based on parameters identification. Phys Lett A 2001;256:148-52.

[6] Fuh CC, Tung PC. Controlling chaos using differential geometric method. Phys Rev Lett 1995;75:2952-5.

[7] Sanchez EN, Perez JP, Martinez M, Chen G. Chaos stabilization: an inverse optimal control approach. Latin Amer Appl Res: Int J 2002;32:111-4.

[8] Lu J, Xie J, Lü J, Chen S. Control chaos in transition system using sampled-data feedback. Appl Math Mech, in press.

[9] Cao YJ. A nonlinear adaptive approach to controlling chaotic oscillators. Phys Lett A 2000;270:171-6.

[10] Lü J, Chen G. A new chaotic attractor coined. Int J Bifurcat Chaos 2002;12:659-61.

[11] Lü J, Chen G, Zhang S. Dynamical analysis of a new chaotic attractor. Int J Bifurcat Chaos 2002;12:1001-15.

[12] Lü J, Chen G, Zhang S. The compound structure of a new chaotic attractor. Chaos, Solitons \& Fractals 2002;14:669-72.

[13] Lorenz EN. Deterministic non-periodic flows. J Atmos Sci 1963;20:130-41.

[14] Stewart I. The Lorenz attractor exists. Nature 2000;406:948-9.

[15] Chen G, Ueta T. Yet another chaotic attractor. Int J Bifurcat Chaos 1999;9:1465-6.

[16] Vaněcek A, Čelikovský S. Control systems: from linear analysis to synthesis of chaos. London: Prentice-Hall; 1996.

[17] Yu Y, Zhang S. Controlling uncertain Lü system using backstepping design. Chaos, Solitons \& Fractals 2003;15:897-902.

[18] Zhou Z. A new chaotic anti-control model - Lü system. J. Xianning Teachers College 2002;3:19-21. 